\title{
CAMBIOS DE USOS DEL SUELO Y ALTERACION DE PAISAJES EN EL VALLE DE CANAL ROYA (prov. Huesca)
}

\author{
Luis Antonio CANCER POMAR \\ Universidad de Zaragoza
}

\begin{abstract}
Resumen: La organización territorial y productiva tradicional del Pirineo, mantenida con escasas variaciones hasta hace pocas décadas, concentró sus actividades en determinados valles y permitio que amplios sectores permaneciesen casi intactos. Este estatus ha cambiado -o está en vías de hacerlo- en los áltimos años, al ubicarse o proyectarse numerosas actividades con notable incidencia espacial en esas areas escasamente alteradas. En el presente artículo se analiza el caso del valle de la Canal Roya, en el Pirineo Aragonés, sobre el que se han proyectado varios aprovechamientos que, caso de llevarse a cabo, modificarían notablemente, entre otros elementos, su actual configuración paisajística.
\end{abstract}

\begin{abstract}
The traditional territorial and productive organization in the Pyrenees, wich has been preserved with few variations until a few decades ago, centered its activities in certain valleys and alloved large extensions to remain virtually intact. This situation has changed -or, rather, is starting to change-in the last few years as a consequence of the location or planning of plenty of activities with a high physical influence on those hardly altered areas. In this article the case of the Canal Roya valley is analysed, in the Aragonese Pyrenee, a valley where several land developments have been projected wich, were they carried out, would highly modify, among other issues, its current landscape configuration.
\end{abstract}

Sumario: 1.- Actuaciones proyectadas sobre la Canal Roya.- 2.- Planteamiento del problema.3.- Minicentrales e impacto ambiental.- 4.- Minicentral de la Canal Roya.- Bibliografía.

\section{1.- ACTUACIONES PROYECTADAS SOBRE LA CANAL ROYA}

El valle de la Canal Roya, afluente por la izquierda del río Aragón, en el Pirineo de Huesca (fig. 1), está expuesto a dos proyectos de aprovechamiento que, caso de aprobarse, modificarían notablemente determinados componentes de su medio natural y, como consecuencia, alterarían su actual configuración paisajística.

El primero de ellos se refiere a una ampliación de las pistas de esquí de la cabecera del Aragón (Astún-Candanchú) y del Gállego (Formigal) a través de este valle, uniendo las citadas 
estaciones y delimitando una superficie esquiable de gran extensión. Esta idea fue impulsada por el comité de promoción de la candidatura olímpica "Jaca 98", aunque posteriormente se desechó debido al elevado impacto medioambiental que tal actuación acarrearía. A pesar de este abandono del proyecto, no hay que desechar la posibilidad de que se trate simplemente de una dilación momentánea, y que tal idea sea en el futuro reavivada por alguna de las muchas mentes que únicamente conciben el desarrollo turístico del Pirineo mediante el expolio de su mayor riqueza: su medio natural.

El segundo consiste en el aprovechamiento hidroeléctrico del valle por medio de la construcción de una "minicentral" hidroeléctrica. En la actualidad este proyecto está siendo estudiado por los organismos competentes en la materia (Comisión Provincial de Urbanismo de la Diputación General de Aragón, que debe autorizar la licencia de obras previo permiso del ayuntamiento afectado -en este caso, Canfranc-, y la Confederación Hidrográfica del Ebro, que debe decidir sobre la provisión de caudales), quienes lo aprobarán o desecharán.

Al tratarse de un proyecto con posibilidades reales de llevarse a cabo próximamente, a diferencia del expuesto en primer lugar, el análisis posterior se centrará en éste, dejando para otra ocasión el referente a la ampliación de las pistas de esquí.

\section{2.- Planteamiento Del PROBlema.}

Las crecientes necesidades energéticas de las sociedades industriales y postindustriales, y las frecuentes crisis que el sector petrolífero suele presentar, han hecho volver muchas miradas hacia una energía -la hidroeléctrica- limpia, barata y tan apenas sujeta a las fluctuaciones temporales, salvo las derivadas de los ritmos hidrológico-climáticos.

Sin embarrgo, para producir esta energía se necesitan embalsar volúmenes más o menos grandes de agua, se deben construir los denominados "pantanos", $\tan$ frecuentes en casi todo el territorio español.

Los pantanos tradicionales, normalmente de tamaño considerable, provocan, como es sabido, multitud de problemas de todo tipo: económicos y poblacionales, ecológicos y paisajísticos. Esta 
es la razón de que sean ampliamente criticados por muchos grupos sociales, desde significados científicos hasta las cada vez más activas asociaciones ecologistas. La Administración no es ajena a esta polémica y, en razón más o menos directa de ella, en los últimos años se han echado atrás proyectos de acusada embergadura como, por poner un ejemplo significativo, el ambalse de "Lorenzo Pardo" en el río Esera, en la cuenca hidrográfica del Ebro.

En este marco de referencia han aparecido recientemente, de forma especial en el último quinquenio, multitud de proyectos de pequeñas centrales hidroeléctricas, conocidas normalmente con la denominación de "minicentrales", que afectan a buena parte del territorio nacional.

\section{3.- MINICENTRALES E IMPACTO AMBIENTAL.}

Se entiende por minicentral, según la actual legislación del MOPU, aquélla que precise una capacidad de embalse inferior a $100.000 \mathrm{~m}^{3}$ y una presa de menos de $15 \mathrm{~m}$. de altura (ver Real Decreto 1131/88). Por parte de las distintas administraciones (estatal, autonómicas...) y de sus grupos promotores se ha insistido en su bondad, aduciendo las siguientes razones: no inundan grandes superficies, no obligan por lo general a desalojar grupos humanos y, debido a su pequeño tamaño, no provocan impactos ecológicos notables. Por contra, su rentabilidad económica parece asegurada, hasta el punto de que bastantes ayuntamientos afectados quieren explotarlas directamente.

Aún cuando los argumentos anteriores son, en muchos casos ciertos, un análisis objetivo de la cuestión debe ir más allá y debe, en consecuencia, centrarse en los problemas ocasionados por estas instalaciones, que también los hay.

La mayoría de estas minicentrales se proyectan en zonas montañosas, en donde la energía de relieve y los abundantes caudales favorecen la producción hidroeléctrica. A menudo se trata de valles muy poco o nada explotados, casi vírgenes, con grandes valores ecológicos que se pueden ver amenazados -por ejemplo, la fauna acuática por disminución de caudales aguas abajo de la presa- y con paisajes dignos de protección, que se ven, irremediablemente, alterados.

Estas infraestructuras hidroeléctricas constan de una serie de elementos que, básicamente, son los siguientes: 
- Azud y toma de aguas

- Tubería forzada: transporta el agua desde el azud hasta las turbinas de la central

- Central y parque de transformación exterior

- Línea de evacuación de la energía.

Además suele ser necesario construir pistas de acceso hasta la zona de las obras, para transportar la maquinaria y los materiales a utilizar.

Todos estos elementos tienen una incidencia en el paisaje, normalmente negativa, innegable y que convendría analizar en todos los casos con la realización del correpondiente Estudio de Impacto Ambiental. En la actualidad no se obliga a ello en España, ya que sólo las grandes presas deben cumplir este requisito (ver R.D.L. 1302/1986 de 28 de junio, y R.D. 1131/1988 de 30 de septiembre).

\section{4.- MINICENTRAL DE LA CANAL ROYA}

La proyectada minicentral de la Canal Roya se enmarca dentro de un amplio programa de aprovechamiento hidroeléctrico en Aragón. En el año 1987, la Diputación General de Aragón relizó con este fin el "Estudio de Identificación y Evaluación de Pequeñas Centrales Hidroeléctricas existentes y de nueva planta en Aragón", en el que se proponen 68 minicentrales de las que un elevado porcentaje se ubican en el Pirineo, por razones topográficas e hidrológicas obvias.

Seguidamente se va a exponer la situación paisajística actual (o preoperacional, utilizando la terminología más frecuente en los E.I.A.) del valle, antes de efectuar el aprovechamiento proyectado; y posteriormente se analizarán los impactos que éste causaría caso de llevarse a cabo.

Este análisis no pretende, en ningún caso, identificarse con un Estudio de Impacto Ambiental completo, para cuya elaboración se necesitarían conocer con total detalle las características de las obras proyectadas. No obstante, con los datos disponibles se puede realizar una aproximación aceptable al problema. 


\subsection{Análisis de la situación preoperacional.}

\subsubsection{Descripción general.}

\section{a) Aspectos naturales.}

El valle de la Canal Roya presenta las características propias, en prácticamente todos los aspectos considerados, de los paisajes alpinos de media y alta montaña. Las cordilleras pertenecientes a la orogenia alpína, y entre ellas los Pirineos, se caracterizan en primer lugar por su acusada anergía de relieve, que todavía se acentúa más durante le Cuaternario por el efecto de la morfogénesis glaciar. El valle de Canal Roya, tanto por su pertenencia a una cordillera alpina como por su elevada altitud, que lo coloca bajo los efectos de las glaciaciones cuaternarias, presenta como rasgo visual más característico la citada energía de relieve -fuertes desniveles topográficos y altas pendientes-, que además condiciona en buena medida el resto de los caracteres paisajísticos.

La Canal Roya consta de dos unidades morfológico-paisafísticas bien definidas: cabecera/valle medio por un lado y valle bajo, por otro. Las obras de aprovechameinto hidroeléctrico proyèctadas se sitúan en la segunda, con una puntualización: el azud se ubica en el contacto entre ambas, en un sector de transición morfológica (fig., 1).

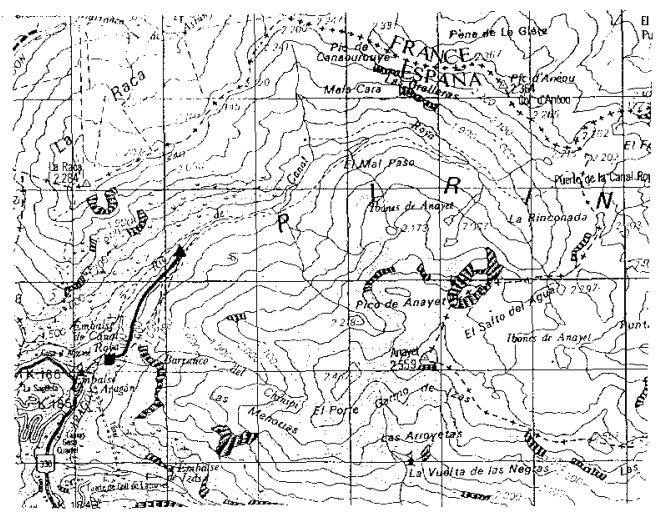

- Amural y pargue de toma de aguas

- Tuberia Comzanal

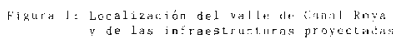

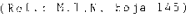


La unidad de cabecera/valle medio se corresponde con un típico valle glaciar en cubeta, con débil incisión fluvial. El perfil longitudinal del río es suave; la vegetación se encuentra representada por pastizal alpino y el grado de naturalidad es muy acusado, pues los únicos elementos antrópicos evidentes son un pequeño refugio, alguna cabaña de pastores y una senda de montaña, sólo apta para circular a pie, que recorre el valle en toda su longitud.

En el sector de contacto entre ambas unidades se producen importantes cambios que afectan a la morfología y al grado de naturalidad. Por un lado, el perfil topográfico transversal deja de ser claramente en cubeta para iniciar una transición a en "V"; por otro, la senda de montaña se convierte en pista forestal apta para el paso de coches.

Las características de la unidad "valle bajo", la realmente afectada por el proyecto, se van a ver con más detenimiento a continuación.

El relieve se encuentra condicionado por la litología de la zona y por el modelado que sobre ésta ha actuado. Se trata de un valle de origen glaciar posteriormente incidido por el barranco de la Canal Roya, que ha labrado un acusado perfil transversal en $\mathrm{V}$. Los materiales aflorantes pertenecen en su mayor parte al Carbonífero -grawacas, pizarras y calizas- y al Permotrías -areniscas y arcillas rojas- (SOLER SAMPERE y PUIGDEFABREGAS, 1972). Precisamente del tono rojizo de estos materiales se toma nombre la canal.

En la actual configuración del relieve tienen una gran importancia los depósitos cuaternarios que tapizan las laderas y el fondo del valle: aluviales -gravas, arenas, limos- y coluviales, estos últimos de gran extensión. Su acusada inestabilidad condiciona cualquier actuación sobre ellos.

Las vertientes se encuentran bastante regularizadas, aunque de forma discontinua presentan canchales a menudo funcionales, normalmente situados en los conos de deyección.

En los sectores de afloramientos calcáreos, como en el punto de ubicación de la presa, las laderas aparecen tapizadas por bloques heterométricos, predominando los de tamaño mediano o grande, caídos por gravedad. Esta caída de bloques se encuentra favorecida por la tectónica plegada y fracturada -hay un elevado número de fallas cruzadas- que afecta a estas calizas. 
La dinámica periglacial actual se manifiesta, además de por la importancia de los depósitos de ladera, en el característico almohadillado que presentan las laderas cubiertas por pastizal.

El perfil en $\mathrm{V}$ antes citado proporciona unas pendientes transversales muy fuertes, a menudo superiores al $100 \%$, lo que unido a la acusada pendiente longitudinal del talwek da como resultado un valle angosto, de horizontes cerrados y de gran energía de relieve. Precisamente en la zona de ubicación de la presa se produce el cambio de perfil longitudinal suave de la unidad de cabecera al fuerte de ésta.

Respecto al cubrimiento vegetal, en este sector el valle presenta un estupendo hayedo-abetal de gran valor ecológico y paisajístico. Se trata de un bosque denso que ha sufrido, al menos en determinados lugares, manejos antrópicos evidentes. Así, en la margen izquierda, a la altura de la presa proyectada, se produce un contacto lateral brusco entre una masa de coníferas y un tramo de pastizal, en forma de contacto lineal, no gradual. En la margen derecha es abundante el pino negro (Pinus uncinata), con orientación general SE.

El río de Canal Roya recorre longitudinalmente el valle; se trata de un característico curso de montaña condicionado tipológicamente por los caracteres topográficos -angostura, acusada pendiente longitudinal-: escasa anchura del cauce, que normalmente no supera los $5 \mathrm{~m}$., y elevada velocidad de las aguas. La notable irregularidad del lecho, tapizado por bloques y cantos rodados, favorece la presencia de remolinos y saltos que dotan de gran dinamismo al discurrir de las aguas.

\section{b) Aspectos antrópicos}

Los usos actuales del suelo, sin ser intensivos, sí que tienen cierta importancia, estando ligados en su mayor parte al sector turístico. En este valle, además de existir un tránsito de montañeros y excursionistas de bastante volumen, ligado al turismo generado por la zona de Canfranc y Candanchú, se localiza un camping con sus correspondientes servicios y un área acondicionada para campamento juvenil.

Otros usos están representados por una pequeña presa de regulación del río, aguas abajo de la ahora proyectada, y algunas corralizas de muros de piedra relacionadas con el ganado extensivo que 
aprovecha los pastos del alto valle de Canal Roya. Por último, aún cuando los manejos forestales antiguos son evidentes, en la actualidad no se aprecian indicios de explotación silvícola reciente.

En cuanto a vías de comunicación, además de varias sendas de muy escasa anchura, sólo aptas para circular a pie y sin tan apenas significado paisajístico, una pista forestal recorre este tramo de valle por su margen derecha.

\section{c) Cromatismo}

Muy variado, por la presencia de bosque denso (color verde oscuro y ocre, según la época del año, por la importante masa caducifolia), pastizal (tono verde claro-amarillento) y afloramientos rocosos que proporcionan, además de los tonos grisáceos habituales, los rojizos de las arcillas y areniscas del Permotrías, sobre todo en la margen derecha.

\subsubsection{Visibilidad}

Se encuentra condicionada principalmente por las características topográficas. En un territorio completamente llano, un objeto o estructura cualquiera se vería claramente hasta que el observador se alejase de él a una distancia en que las correcciones por curvatura de la tierra y refracción de la luz igualasen la altura del objeto. En la Canal Roya, obviamente, estas condiciones no se dan, ya que la energía y sinuosidad del relieve actúan de auténticos cierres visuales, de forma que los objetos o construcciones no son visibles por lo general más allá de unos pocos centenares de metros de su ubicación, por lo que pertenecen a la categoría de "próximos o intraoculares", siguiendo la clasificación de VAN DER HAM (CEOTMA, 1982).

La visibilidad interna del valle, considerando como tal a la percibida desde el interior del mismo, es por lo tanto escasa. Unicamente en su sector más bajo, en la confluencia con el valle del río Aragón, la topografía se abre considerablemente, por lo que aumenta de manera notable esta visibilidad.

La visibilidad externa -desde sectores no pertenecientes a la Canal- sólo es elevada en el tramo de confluencia de este valle con el del Aragón. El resto de su territorio se encuentra 
marginado por cimas de $2.200-2.400 \mathrm{~m}$. que suponen verdaderas divisorias hidrológico-paisajísticas totalmente estancas.

\subsubsection{Fragilidad.}

La fragilidad hace referencia a la capacidad de absorción de elementos extraños. En este sentido, las áreas montañosas resultan muy frágiles, ya que sus cafacterísticas geomorfológicas, de cubrimiento vegetal y antrópicas se encuentran en un equilibrio inestable fácilmente alterable con la introducción de elementos novedosos.

\subsubsection{Valoración}

La valoración del paisaje se va a realizar siguiendo los esquemas propuestos por el MOPU (1989) y por RAMOS (1980) para los estudios ambientales y paisajísticos, analizando diversos parámetros de interés.

- Singularidad. Media, pues la Canal Roya no presenta caracteres inhabituales al resto del Pirineo Central -su área de referencia- salvo la característica coloración rojiza de los afloramientos del Permotrías.

- Diversidad. Alta, por las variaciones geomorfológicas, cromáticas, vegetales y de usos antrópicos existentes en los distintos sectores del valle.

- Calidad. La valoración de la calidad de un paisaje es muy compleja y está sometida a un acusado grado de subjetividad si lo que se pretende es detectar su belleza o fealdad. Una forma de evitar esta valoración basada en los mecanismos psicológicos de los observadores, y por lo tanto sujeta a variaciones de toda índole, consiste en la estimación diferenciada y objetivable de varios parámetros que habitualmente se consideran correctos indicadores de calidad. En las áreas montañosas los dos componentes que normalmente determinan el grado de aceptación de un paisaje son: a) naturalidad, y b) integración antrópica. El mantenimiento de los parajes con un elevado nivel de naturalidad es algo que se valora positivamente. Cuando esto no es posible y la presencia de elementos antrópicos es notable, se considera de forma 
positiva el que los mismos se encuentren correctamente integrados en el entorno natural.

En la Canal Roya el grado de naturalidad es alto, aunque la existencia de los aprovechamientos expuestos en el apartado 4.1.1. b no permite alcanzar la valoración de "muy alto" de pareas cercanas. Respecto a la integración antrópica, se considera igualmente alta, por tratarse en general de construcciones bien integradas y que mantienen la tipología tradicional de la zona.

- Valoración global. En el cuadro anexo aparece la valoración de cada uno de los parámetros considerados y, como síntesis de ello, la valoración global paisajística:

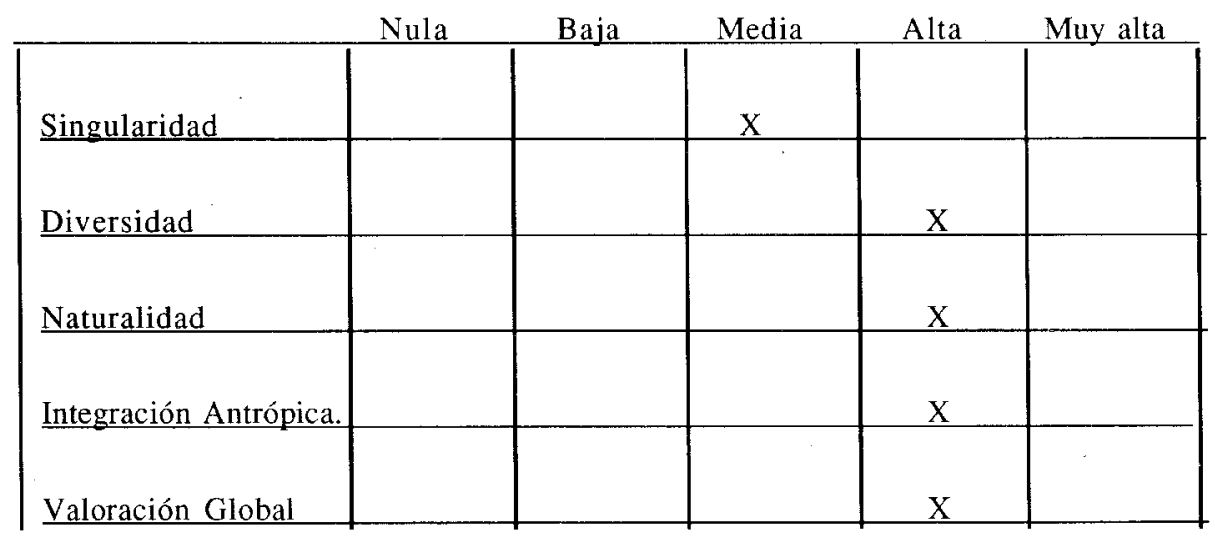

\subsection{Análisis de impactos}

En este apartado se va a analizar qué compoenente con incidencia paisajística quedarían afectados por las obras proyectadas.

\subsubsection{Descripción}

Impacto 1: alteración de las formas de relieve.

Provocado por la tubería forzada, que obligará a excavar una zanja, en la que irá enterrada, de más de $1.300 \mathrm{~m}$. de longitud, en la margen izquierda del valle. La excavación de terrenos inestables, 
como son los depósitos de ladera que tapizan todo el sector, puede acarrear movimientos en masa de los mismos, deslizamientos por gravedad como efecto de la ruptura del perfil de equilibrio de una ladera de fuertes pendientes.

El resto de los elementos integrantes del aprovechamiento hidroeléctrico no obligan tan apenas a realizar movimientos de tierras.

Impacto 2: Denudación de la cubierta vegetal.

Provocado por la tubería forzada, la línea aérea de evacuación de la energía, el azud y el conjunto central-parque exterior.

La apertura de la zanja donde irá enterrada la tubería forzada obligará a talar un pasillo de considerables dimensiones en un bosque mixto de gran variedad pero de extensión reducida. El difícil acceso a esta zona de fuertes pendientes hace prever que las talas no se limitarán exclusivamente al corredor ocupado por la tubería, sino que afectarán también a terrenos aledaños.

La línea de A.T. de evacuación de la energía, de casi $400 \mathrm{~m}$. de longitud, precisará la tala de un pasillo de $7 \mathrm{~m}$. de ancho a lo largo de todo su recorrido.

El azud afectará a un sector cubierto exclusivamente por pastizal (Bromus erectus y Festuca rubra), de escasa extensión.

La ubicación del conjunto central-parque de transformación en un terreno explanado previamente obligará a eliminar su cubierta vegetal, aunque la superficie afectada será muy reducida.

\section{Impacto 3: Modificaciones cromáticas.}

Provocado por la presa, la tubería forzada, el conjunto central-parque exterior y la línea aérea de A.T.

La presa está proyectada en hormigón, material blanquecino que supondrá un elemento visual extraño en relación a las tonalidades verdoso-amarillentas y verde oscuras pertenecientes 
respectivamente al pastizal y a las coníferas que tienen su punto de contacto en las inmediaciones de aquélla.

La tubería forzada introducirá un pasillo de coloración terrosa en una masa intensamente verde durante la primavera y el verano.

El color blanco del edificio de la central (proyectado en hormigón) y los tonos metálicos del parque exterior y de la línea aérea de A.T. modificarán el actual cromatismo de su entorno, básicamente verde.

Impacto 4: Alteraciones de la cuenca visual.

Debidas a la presencia de elementos nuevos más o menos visibles.

La presa se ubicará en un sector rehundido entre dos hombreras glaciares, que dificultan la visión del fondo del valle por lo que la visibilidad interna resultará poco afectada. No ocurrirá lo mismo con la tubería forzada, pues al discurrir a media ladera será perfectamente visible desde cualquier sector. Acusada visibilidad interna presentará también el conjunto central-parque exterior, ubicado en un amplio -para lo que es normal en la Canal-fondo de valle.

La línea de A.T. discurrirá por el tramo de contacto entre la Canal Roya y el valle del Aragón, en un sector muy abierto topográficamente situado entre dos unidades paisajísticas diferentes, por lo que tanto la visibilidad interna como la externa serán alteradas.

Impacto 5: Aparición de estructuras antrópicas novedosas.

De notable significado en un valle poco humanizado.

\subsubsection{Valoración}

La valoración de impactos se ha realizado a partir de la consideración de varios factores que presentan, cada uno, significado por sí mismos y que conjuntamente permiten predecir la magnitud de aquéllos (ver cuadro anexo). 
Esta valoración debería conducir a la calificación final de cada impacto (utilizando adjetivos del tipo "nulo", "aceptable", "crítico", etc.). Sin embargo, la escasa información disponible para la elaboración del presente artículo impide -so pena de asumir un riesgo excesivo- realizar una calificación precisa. De todas formas, sí que se puede adelantar que el elemento paisajístico más afectado será la vegetación, por las talas necesarias para la ubicación de la tubería forzada en un bosque de gran valor ecológico y perceptual. Afecciones acusadas serán también las derivadas de la instalación de la línea aérea de A.T., que modificará negativamente la visibilidad de un sector de topografía abierta, y la aparición de nuevas estructuras antrópicas en un valle poco alterado hasta la fecha.

\begin{tabular}{|c|c|c|c|c|c|}
\hline & Impacto 1 & Impacto 2 & Impacto 3 & Impacto 4 & Impacto 5 \\
\hline Carácter & Negativo & Negativo & Negativo & Negativo & Negativo \\
\hline Duración & Permanente & Permanente & Permanente & Permanente & Permanentte \\
\hline Proyección & & & & & \\
\hline temporal & Corto plazo & Corto plazo & Corto plazo & Corto plazo & Corto plazo \\
\hline Proyección & & & & & \\
\hline espacial & Puntual & Local & Local & Local & Local \\
\hline $\begin{array}{l}\text { Afección a } \\
\text { singularidades }\end{array}$ & No & Si & $\mathrm{Si}$ & No & No \\
\hline $\begin{array}{r}\text { Probabilidad de } \\
\text { ocurrencia }\end{array}$ & Media & Alta & Alta & Alta & Alta \\
\hline
\end{tabular}

\section{BIBLIOGRAFIA}

CEOTMA (1982): Guía para la elaboración de estudios del medio físico. Contenido y metodología. MOPU, madrid.

DIPUTACION GENERAL DE ARAGON (1987): Estudio de identificación y evaluación de pequeñas centrales existentes y de nueva planta en Aragón. (Inédito).

MOPU (1989): Guías metodológicas para la elaboración de estudios de Impacto Ambiental. Madrid.

RAMOS, A. (1980): El estudio del paisaje. Cátedra de Planificación y Proyectos. E.T.S.I.M. de Madrid. 
SOLER SAMPERE, M. y PUIGDEFABREGAS, C. (1972): Esquema litológico del Alto Aragón Occidental. Rev. Pirineos, $\mathrm{n}^{0} 125$, C.S.I.C. Jaca. 